\title{
Abnormally invasive placenta: an overview of diagnosis and management options
}

\author{
Parveen Rajora $^{1 *}$, Amanjot Singh ${ }^{2}$
}

\begin{abstract}
${ }^{1}$ Department of Obstetrics and Gynecology, Guru Gobind Singh Medical College, Faridkot, Punjab, India
${ }^{2}$ Department of Anesthesia, Guru Gobind Singh Medical College, Faridkot, Punjab, India
\end{abstract}

Received: 24 August 2017

Accepted: 25 September 2017

\author{
*Correspondence: \\ Dr. Parveen Rajora, \\ E-mail: parveenrajora@yahoo.com
}

Copyright: $($ ) the author(s), publisher and licensee Medip Academy. This is an open-access article distributed under the terms of the Creative Commons Attribution Non-Commercial License, which permits unrestricted non-commercial use, distribution, and reproduction in any medium, provided the original work is properly cited.

\begin{abstract}
Background: Abnormally invasive placenta, also known as morbidly adherent placenta, is a broad term that describes abnormal adherence of placenta to the underlying myometrium. Clinical risk factors include placenta previa and prior uterine surgery, including caesarean delivery. The diagnosis and management of women at risk is not only based on clinical parameters, but also driven by imaging, namely ultrasound and more recently magnetic resonance (MRI) imaging.

Methods: This is a retrospective analysis of 10 cases of abnormally invasive placenta undertaken at Guru Gobind Singh Medical College and Hospital, Faridkot.

Results: Hysterectomy done in six cases and uterus was preserved in four cases. Foetal outcome was on average side. Four foetal losses noted two because of prematurity and two due to excessive blood loss admitted with intra uterine foetal death. Two babies needed NICU care but successfully discharged.

Conclusions: Newer approaches should be considered investigational until larger prospective series to become available, until then hysterectomy should remain the stay of treatment specially when the family is complete and there is life threatening Haemorrhage.
\end{abstract}

Keywords: Abnormally invasive placenta, Hysterectomy, MRI, Ultrasonography

\section{INTRODUCTION}

Abnormally invasive placenta, also known as morbidly adherent placenta, is a broad term that describes abnormal adherence of placenta to the underlying myometrium. Depending on the depth of invasion, it is further defined as placenta accrete, placenta increta and placenta percreta.

The term "accrete" is the umbrella term most commonly used to refer to all of these conditions. The term "abnormally invasive placenta (AIP) was introduced in 2013 and defined as placenta that cannot be removed spontaneously or manually without causing severe bleeding. Properly the chorionic villi of the placenta accrete grow into the basal decidua within the uterus, whereas these villi in the placenta increta and the placenta percreta go in to the musculature and the myometrium of the uterus respectively. Then, infiltration in serosa and even in the neighbouring organs such as the urinary bladder and bowel can occur so serious complication may arise. ${ }^{1}$ Clinical risk factors include placenta previa and prior uterine surgery, including caesarean delivery.

The incidence of AIP has increased with the rising number of caesarean delivery (CD) current estimates 
indicate a $25 \%$ to $50 \%$ incidence of AIP in patients with placenta previa and prior CD. ${ }^{2}$

Complications of AIP include massive haemorrhage, damage to the uterus, bladder, ureter and bowl and often caesarean Hysterectomy $(\mathrm{CH})$ to control bleeding. Prenatal diagnosis of AIP can help minimize the complication rate by enabling a surgeon to plan for the type of resources needed at the time of delivery. These resources include obstetric anaesthesia appropriate surgical expertise, possible interventional radiology for uterine artery embolization and post-operative intensive care. $^{3}$

The diagnosis and management of women at risk is not only based on clinical parameters, but also driven by imaging, namely ultrasound and more recently magnetic resonance (MRI) imaging. Prenatal ultrasonography (USG) is formerly considered as the routine diagnostic standard examination, but foetal MRI is emerging as an alternative and complimentary method. ${ }^{4}$

The traditional approach to management has been entirely surgical namely en-bloc-hysterectomy with close physician support of circulatory indices, often in the intensive care unit. Maternal morbidity is known to be significant and cases of maternal mortality are very occasionally reported.

Nevertheless, in recent years, more conservative management strategies are being examined to potentially minimize this surgical morbidity and to preserve women fertility like by leaving the placenta in situ at the time of C.D. and allowing later resorption or expulsion to occur. Furthermore, methotrexate may be administered to enhance resolution.

Other surgical options have also been performed to minimize the blood loss including proximal ligation or embolization of the internal iliac artery (IIA) or uterine artery (UA).
The objective of the study is to illustrate our experience with AIP and to discuss the optimal diagnosis and management modalities for this disease with joint efforts of other authors.

\section{METHODS}

This is a retrospective analysis of 10 cases of abnormally invasive placenta undertaken at Guru Gobind Singh Medical College and Hospital Faridkot. For the purpose of description, the term Abnormal invasive placenta (AIP) is used to represent all types of morbidly adherent placenta informed written consent was obtained from the participants. Cases were only included if they encomposed AIP confirmed histopathologically or clinically during surgery. For each case the following information was registered: gestational age at delivery, method of pre-natal diagnosis, existence of a preoperative plan for surgery whether it was an elective or acute caesarean section, indication for delivery in case of emergency CS, history of any previous uterine surgery, Surgical procedures adopted, post-operative complications, amount of blood loss in $\mathrm{ml}$ or transfusion required in unit.

\section{RESULTS}

Patients demographics are shown in Table 1. Among the ten patients the mean age was 35 years. All women had risk factors such as placenta previa, previous caesarean delivery and/or dilatation and curettage for abnormally invasive placenta.

There were 5 patients diagnosed ante natelly as placenta accreta with placenta previa remaining 5 were diagnosed clinically when manual removal of the placenta was impossible because of the absence of complete cleavage plane between the placenta and the uterus. These were the cases who were immediately taken for surgery due to intractable bleeding per vagina.

Table 1: Demographic profile of patients $(\mathrm{N}=10)$.

\begin{tabular}{|c|c|c|c|c|c|}
\hline Gravidity & Age & PoG in weeks & No. of previous LSCS & D and C & History of Myomectomy \\
\hline G3 & 34 & 36 & 2 & 0 & \\
\hline G4 & 30 & 33 & 3 & 0 & \\
\hline G3 & 32 & 18 & 1 & 1 & 1 \\
\hline $\mathrm{G} 2$ & 34 & 22 & 1 & 0 & \\
\hline G4 & 35 & 36 & 2 & 1 & \\
\hline G3 & 30 & 37 & 2 & 0 & \\
\hline G3 & 29 & 34 & 1 & 1 & 1 \\
\hline G4 & 28 & 33 & 1 & 2 & \\
\hline G3 & 33 & 35 & 2 & 0 & \\
\hline G3 & 30 & 36 & 2 & 0 & \\
\hline
\end{tabular}

Number $(\mathrm{N})=10 ; \mathrm{PoG}=$ Period of gestation; $\mathrm{D}$ and $\mathrm{C}=$ Dilatation and Curettag 
All of the study ten cases are of abnormally invasive placenta with placenta previa confirmed antenatally or clinically during surgery or by histopathologically in hysterectomised uterus. Four patients were diagnosed by ultrasonography and 2 of them confirmed by MRI also. Other two patients refused for MRI due to economic reasons or by fear. Five women underwent planned caesarean delivery at the mean gestation age of 34 weeks (31-37 weeks) Two women underwent hysterectomy at 18 weeks and 22 weeks respectively. Hysterectomy was required in six of the ten patients. In three patients Hysterectomy was planned as the initial procedure. Tubal ligation was also done in three preserved uteruses except one when hysterotomy done at 18 weeks. We took every measure to reduce blood loss like uterine artery ligation (done in 6 cases), internal iliac artery ligation (done in 4 cases) uterine packing was required in 2 cases. Mean maternal blood loss during planned caesarean delivery was 1.5-2 L. It was slightly higher in emergency caesarean Hysterectomy cases. Placenta was left in uterus for spontaneous resolution in 2 cases. Methotrexate was given to one patient along with folinic acid rescue.

Table 2: Mode of diagnosis.

\begin{tabular}{|l|l|}
\hline Mode of diagnosis & Number of patients \\
\hline USG & 4 \\
\hline MRI & 2 \\
\hline Clinically during surgery & 6 \\
\hline Histopathologically & 6 \\
\hline
\end{tabular}

In the present series, the uterus was preserved in four cases. Two of the Hysterotomy cases and two who had focal invasion (Table 3).

Table 3: Surgical procedure followed.

\begin{tabular}{|l|l|}
\hline Surgical procedure & N \\
\hline Hysterectomy & 6 \\
\hline Uterus preserved & 4 \\
\hline Placenta left in situ & 2 \\
\hline Methotrexate & 1 \\
\hline Tubal ligation & 3 \\
\hline UAL & 6 \\
\hline IIAL & 4 \\
\hline Uterine packing & 2 \\
\hline UAL: Uterine artery ligation; IIAL: Internal iliac artery ligation
\end{tabular}

Table 4: Complications.

\begin{tabular}{|l|l|}
\hline Complication & No of patients \\
\hline Haemorrhage & 6 \\
\hline Bladder injury & 2 \\
\hline GUT injury & 1 \\
\hline Sepsis & 3 \\
\hline Need for blood transfusion & 10 \\
\hline Hospital stay $>15$ days & 3 \\
\hline
\end{tabular}

Fortunately, no maternal mortality was recorded but these patients had suffered a lot of morbidity as illustrated in Table 2. No rectovaginal fistula was reported.

Foetal outcome was on average side. Four foetal losses noted two because of prematurity and two due to excessive blood loss admitted with intra uterine foetal death. Two babies needed NICU care but successfully discharged.

\section{DISCUSSION}

Demographic profile and risk factors pertaining to AIP like, old age, high gravidity, previous uterine surgery like $\mathrm{CS}, \mathrm{D}$ and C, myomectomy is in accordance with other authors. AIP has been reported to have up to $7 \%$ mortality rate with lot of intra operative and postoperative complications. In the present study most common was hemorrhage requiring blood transfusion, bladder injury and difficulty encountered during surgery due to adhesions and invasions. Fortunately, no maternal mortality occurs. ${ }^{1}$

Antenatal diagnosis of the disease has a great impact on the final outcome. In the present series antenatal diagnosis was make possible in four patients, as our is tertiary catering hospital and mainly cater referral cases. Diagnosis of AIP is possible with routine USG complimented with MRI in equivocal cases. Detection rate of USG ranges from $33 \%$ to $100 \%$. Loss of Hypoechoiec retro placental myometrial zone, presence of placental lacunae, disruption of the posterior bladder uterine interface and in case of placenta percreta presence of focal exophytic masses are the diagnostic criteria. Doppler shows turbulent blood flow into the surrounding tissues, subplacental vascularity vessels are more heterogenous in size and less uniformly distributed MRI appears to add information only when the placenta is not well depicted on USG for example in posterior placenta accrete. Prenatal diagnosis is crucial in order to plan delivery and to reduce complication. ${ }^{4}$

Management of AIP requires a multidisciplinary team approach. Surgical management strategies for AIP generally include the extirpative approach (attempt to remove placenta) $\mathrm{CH}$ and conservative management (with or without partial resection of placenta) Nevertheless, the best policy remains a matter of debate optimal timing of delivery remain controversial. ${ }^{6,7}$ However some recommend a planned caesarean/hysterectomy. In our elective cases, we have tried to cross 36 weeks POG at least. In planned CS abdomen was opened according to previous scar but uterine scar was classical caesarean scar careful observation are mandatory in women with preserved uterus with respect to possible increased risk of uterine rupture in future pregnancy. To overcome this tubal ligation done in three out of four preserved uteruses. 
However, some author found transverse fundal incision method more superior to the conventional method with respect to reduction of blood loss. ${ }^{8}$

Successful management of AIP requires prevention and control of bleeding. Specific techniques that have been described for the management of AIP retrospective reviews and case reports include uterine and vaginal packing with gauze, balloon tamponate, the B Lynch suture, vertical compression suture and suturing an inverted lip of cervix over the bleeding site. ${ }^{5}$ Dubos et al reported that temporary balloon occlusion of the bilateral iliac artery is method to control bleeding. ${ }^{9}$ This method is supported by some authors but found ineffective by others. We did internal iliac artery (IIA) and uterine artery (UA) ligation in sex patients and found it decreases intra operative blood loss to some extent. Ligation of hypogastric artery is known to diminish utero placental blood flow by up to $30 \%$. Qinghua Wu has come up with newer technique of balloon occlusion of the infrarenal abdominal aorta during CS as efficacy of occlusion of IIA and UA is limited by rapid recruitment of an extensive collateral system in the pelvis. ${ }^{10}$ Ricardo has successfully used polidocanol, a sclerosing agent in eleven patients to decrease blood loss. ${ }^{11}$ Some found it unsafe due to the possibility of allergic reaction, micro embolism associated strokes, transient ischemic attacks and a long contra indication list. ${ }^{12}$

As $20 \%$ of women with AIP are primiparous, it is essential to preserve the future fertility of women, expectant and medical management plays a great role in this aspect. Previously a more conservative treatment aiming at uterine rescue was followed, based upon manual removal of as much placenta as possible. Fox et al reported that $25 \%$ of the women died during this treatment. ${ }^{13} \mathrm{We}$ were fortunate to save the patient when this technique was used in un diagnosed AIP resulting in massive hemorrhage even after IIL and UAL. We left the placenta in situ in two cases in which hysterotomy was done. One case with 18 weeks POG spontaneous expulsion of placenta occurs after 3 weeks and in one with 28 weeks, we successfully tried methotrexate with folinic acid rescue regime, under antibiotic cover for sepsis. In the present series we preserved four out of nine uterus. When there is no hemorrhage and infection, placenta left in situ can be followed by color Doppler for cessation/regression of blood into myometrial component and attempt can be made after to evacuate or remove the placenta, using methotrexate for spontaneous resorption is debatable. Currently there is insufficient evidence on its efficacy and safety and there are no clear guide lines available on dose regime. ${ }^{14}$ Other conservation management can be resection of the myometrium and repairs as described by Palacios et al. ${ }^{15}$ Though a fully conservative management is possible as suggested by Welsh et al still patient should be warned of the bleeding and infections related risk. ${ }^{16}$
We did planned $\mathrm{CH}$ in 3 cases and emergency $\mathrm{CH}$ in 3 cases. The outcome was much better in planned $\mathrm{CH}$. To some extent our surgical strategy was similar to Matsubara S like ligation of IIA and UA, holding of cervix, filling the bladder for identification of the bladder top and to check the integrity of bladder. ${ }^{17}$ We placed uretral stunts in two cases like Tam which really helped to reduce ureteric injury. ${ }^{18}$

The decision to perform a caesarean hysterectomy or conservative management is based on the extent of infiltration, patients hemodynamic condition, her desire to remain fertile. The high risk of infection and hemorrhage must not be overlooked should conservative treatment be chosen.

\section{CONCLUSION}

There is a need to maintain a higher index of suspicion of AIP in high risk women and preparations for delivery should be made accordingly. Antenatal diagnosis probably affords the best chances of reducing blood loss and morbidity over all. Newer approaches should be considered investigational until larger prospective series to become available. More over radiological interventions are not available at all centres, until then hysterectomy, rather planned hysterectomy should remain the stay of treatment specially when the family is complete and there is life threatening haemorrhage.

\section{Funding: No funding sources \\ Conflict of interest: None declared}

Ethical approval: The study was approved by the Institutional Ethics Committee

\section{REFERENCES}

1. Chantraine F, Langhoff-Roos J. Abnormally Invasive placenta-AIP. Awareness and pro-active management is necessary. Acta Obstet Gynecol Scand. 2013;92:369-71.

2. Fitzpatrick KE, Sellers S, Spark P, Kurinczuk JJ, Brocklehurst P, Knight M. Incidence and risk factors for placenta accreta/increta/percreta in the UK: a national case-control study. PLOS ONE. 2012;7(12).

3. Gielchinsky Y, Rojansky N, Fasouliotis SJ, Ezra Y. Placenta accrete-summary of 10 years: a survey of 310 cases. Placenta. 2002;23:210-4.

4. Dwyer BK, Belogolovkin V, Tran L, Rao A, Carroll I, Barth R, et al. Prenatal diagnosis of placenta accreta. J Ultrasound Med. 2008;27:1275-81.

5. Royal College of Obstetricians and Gynaecologists. Placenta praevia, placenta praevia accreta and vasa praevia: diagnosis and management. Green top Guideline no 27 London UK 10. 2011;3:1-26.

6. Kayem G, Davy C, Goffinet F, Thomas C, Clement D, Cabrol D. Conservative versus extirpative management in cases of placenta accreta. Obstet Gynecol. 2004;104:531-6. 
7. Grace Tan SE, Jobling TW, Wallace EM. Surgical Management of placenta accreta: a 10-year experience. Acta Obstet Gynecol Scand. 2013;92:445-50.

8. Nishida R, Yamada T, Akaishi R, Kojima T. Usefulness of transverse fundal incision method of cesarean section for women with placentas widely covering the entire anterior uterine wall. J Obstet Gynaecol Res. 2013;39:91-5.

9. Dubois J, Garel L, Grignon A, Lemay M, Leduc L. Placenta percreta: balloon occlusion and embolization of the internal iliac arteries to reduce intraoperative blood losses. Am J Obstet Gynecol 1997;176(3):723-6.

10. Wu Q, Liu Z, Zhao X, Liu C, Wang Y, Chu Q, et al. Outcome of pregnancies after balloon occlusion of the infrarenal abdominal aorta during caesarean in 230 patients with placenta praevia accreta. Cardiovascular Interventional Radiol. 2016;39(11):1573-9.

11. Reyes RM, de Ávila RC, Vázquez MD, Monteagudo CA, Zerón HM. Sclerotherapy with $6 \%$ polidocanol solution in patients with placenta accreta. Taiwanese J Obstet Gynecol. 2016;55(5):654-8.

12. Guex, JJ. Complications and side-effects of foam sclerotherapy. Phlebol. 2009;24(6): 270-4.

13. Fox H. Placenta accreta 1945-1969. Obstetrical Gynecological Survey. 1972;27(7):475-90.
14. Dasari P, Venkatesan B, Thyagarajan C, Balan S. Expectant and medical management of placenta increta in a primiparous woman presenting with postpartum haemorrhage: The role of imaging. J Radiol Case Reports. 2010;4(5):32.

15. Palacios Jaraquemada JM, Pesaresi M, Nassif JC, Hermosid S. Anterior placenta percreta: surgical approach, hemostasis and uterine repair. Acta Obstet Gynecol Scand. 2004;83:738-44.

16. Welsh AW, Ellwood D, Carter J, Peduto AJ, Veelago J, Bennett M. Opinion: integration of diagnostic and management perspectives for placenta accrete. Aust N Z J of Obstet Gynecol. 2009;49:578-87 .

17. Matsubara S, Kuwata T, Usui R, Watanabe, Izumi A, Ohkuchi A, et al. Important surgical measures and techniques at cesarean hysterectomy for placenta previa accreta. Acta Obstet Gynecol Scand. 2013;92:372-7.

18. Tam Tam KB, Dozier J, Martin JN Jr. Approaches to reduce urinary tract injury during management of placenta accreta, increta, and percreta: a systematic review. J Matern Fetal Neonatal Med. 2012;25(4):329-34.

Cite this article as: Rajora $\mathrm{P}$, Singh $\mathrm{A}$. Abnormally invasive placenta: an overview of diagnosis and management options. Int J Reprod Contracept Obstet Gynecol 2017;6:5013-7. 